\title{
Violência ocupacional sofrida por enfermeiros no contexto da Atenção Básica
}

\author{
Occupational violence suffered by nurses in the context of Primary Care \\ Violencia ocupacional que sufren enfermeras en el contexto de la Atención Primaria \\ Monique Góes Alexandre ${ }^{1 *}$, Monik Rocha de Souza ${ }^{1}$, Luiz Gonzaga Lopes Barroso Neto', Jabneela \\ Vieira Pereira Vetorazo'.
}

\section{RESUMO}

Objetivo: Analisar o impacto da violência ocupacional sobre a saúde profissionais da enfermagem que atuam na Atenção Primária de Saúde (APS). Métodos: Trata-se de uma revisão integrativa da literatura, qualitativa/qualitativa, realizada entre os meses de Julho 2021 a Setembro 2021, nas bases de dados da Acervo+, Literatura Latino-Americana e do Caribe em Ciências da Saúde (LILACS), Literatura Internacional em Ciências da Saúde (PUBMED), na Biblioteca Eletrônica Científica Online (Scielo) e Biblioteca Virtual em Saúde (BVS), a partir da inclusão de artigos científicos disponíveis na íntegra, em livre acesso, publicados no período entre 2016 a 2021, nos idiomas português, inglês e espanhol, bem como aqueles que, após leitura do título e resumo, abordassem aspectos relacionados à temática do estudo. Resultados: Encontraram-se 108 artigos nas bases de dados, e após a aplicação dos critérios de exclusão, selecionados 18 artigos para subsidiar a revisão de literatura. Considerações finais: Os impactos decorrentes da violência ocupacional no profissional da enfermagem na APS são de natureza psicológica, emocional e física, tias como: danos físicos, insegurança, ansiedade manifestações emocionais, transtornos e distúrbios psíquicos, depressão, sofrimento, desejo de abandonar a profissão. Além disso afeta diretamente o desempenho e a assistência de enfermagem.

Palavras-chave: Enfermagem, Violência ocupacional, Atenção primária a saúde.

\begin{abstract}
Objective: To analyze the impact of occupational violence on the health of nursing professionals who work in Primary Health Care (PHC). Methods: This is an integrative qualitative/qualitative literature review, carried out from July 2021 to September 2021, in the databases of Acervo+, Latin American and Caribbean Literature in Health Sciences (LILACS), Literature International in Health Sciences (PUBMED), in the Online Scientific Electronic Library (Scielo) and Virtual Health Library (VHL), from the inclusion of scientific articles available in full, with free access, published in the period between 2016 to 2021, in the Portuguese, English and Spanish languages, as well as those that, after reading the title and abstract, addressed aspects related to the study theme. Results: 108 articles were found in the databases, and after applying the exclusion criteria, 18 articles were selected to support the literature review. Final considerations: The impacts resulting from occupational violence on nursing professionals in PHC are psychological, emotional and physical, such as; physical damage, insecurity, anxiety, emotional manifestations, mental disorders and disorders, depression, suffering, desire to leave the profession. Furthermore, it directly affects nursing performance and care.
\end{abstract}

Key words: Nursing, Occupational violence, Primary health care.

\footnotetext{
${ }^{1}$ Centro Universitário Aparício Carvalho (FIMCA), Porto Velho - RO.

*E-mail: monickrocha23@gmail.com
} 


\section{RESUMEN}

Objetivo: Analizar el impacto de la violencia laboral en la salud de los profesionales de enfermería que laboran en Atención Primaria de Salud (APS). Métodos: Se trata de una revisión integradora cualitativa / cualitativa de la literatura, realizada entre julio de 2021 y septiembre de 2021, en las bases de datos de Acervo +, Literatura Latinoamericana y del Caribe en Ciencias de la Salud (LILACS), Literatura Internacional en Ciencias de la Salud (PUBMED), en el Biblioteca Electrónica Científica en Línea (Scielo) y Biblioteca Virtual en Salud (BVS), a partir de la inclusión de artículos científicos disponibles en su totalidad, con acceso gratuito, publicados en el período comprendido entre 2016 y 2021, en los idiomas portugués, inglés y español, así como los que, luego de la lectura del título y el resumen, abordaron aspectos relacionados con la temática de estudio. Resultados: se encontraron 108 artículos en las bases de datos, y luego de aplicar los criterios de exclusión, se seleccionaron 18 artículos para sustentar la revisión de la literatura. Consideraciones finales: Los impactos derivados de la violencia ocupacional en los profesionales de enfermería de la APS son psicológicos, emocionales y físicos, tales como: daño físico, inseguridad, ansiedad, manifestaciones emocionales, trastornos y trastornos mentales, depresión, sufrimiento, deseo de dejar la profesión. Además, afecta directamente el desempeño y los cuidados de enfermería.

Palabras clave: Enfermería, Violencia laboral, Atención primaria de salud.

\section{INTRODUÇÃO}

A violência ocupacional se caracteriza por abusos ou ameaças físicas no ambiente laboral, em situações relacionadas ao seu trabalho, levando as vítimas ao sentimento de medo, insegurança, gerando risco para sua saúde e bem-estar. Trata-se de um tipo de violência praticada em todo o mundo, e os profissionais de saúde também podem ser vítimas da mesma em seu cotidiano laboral, sendo que mundialmente, 70 a $80 \%$ das equipes de enfermagem vivenciaram um ou mais casos de violência (TSUKAMOTO SAS, et al., 2019).

Nesse contexto, o ambiente de trabalho vem sendo afetado grandemente pelo avanço da violência. Conforme Tavares DS, et al. (2021), a violência ocupacional é toda ação que abrange o comportamento de uma pessoa contra outra profissional que leve à agressão, ofensa, prejuízo e humilhação no decorrer de suas atividades laborais. Os autores afirmam ainda que o número de violências cometidas e sofridas por enfermeiros é grande, especialmente a verbal, sendo que muitos são desprezados, tratados de forma inapropriada, vítimas de deboches, hostilidades por meio de olhares e insultos, dentre outras formas desrespeitosas de tratamento.

No estudo de Bernardes MLG, et al. (2020), sobre o tema, verificou dentre $88,9 \%$ dos entrevistados (trabalhadores de enfermagem) disseram ter sofrido violência ocupacional: $38 \%$ sofreu abuso verbal; $25,4 \%$ sofreu assédio moral; $11 \%$ sofreram violência física; $9,1 \%$ sofreu assédio sexual; $5,4 \%$ sofreu discriminação racial. Os índices são preocupantes, ainda mais quando se constata que $90 \%$ destes profissionais afirmam a violência ocupacional que foram acometidos poderia ter sido evitada.

Diante desses fatores, o profissional que sabe conduzir o gerenciamento de crises e conflitos, pode ter o seu trabalho valorizado, especialmente em contextos que demandam a administração de interesses díssonos e inflamáveis. São situações onde o paciente, e sua família, estão preocupados, angustiados o que, aliado ao já comum estresse do cotidiano, pode gerar relacionamentos não muito saudáveis, para ambas as partes. A literatura aponta que o profissional que sofre esse tipo de violência tende ter problemas no desempenho de suas funções, não atendendo os usuários a contento (TEIXEIRA NL, et al., 2018).

A enfermagem que atua em unidades de urgência e emergência hospitalar se apresentam mais expostos a violência laboral, quando comparadas a APS (FREITAS RJM, et al., 2017). Neste sentido, toma-se por base uma pesquisa desenvolvida por Freitas RJM, et al. (2017) envolvendo profissionais da enfermagem, a qual apontou que atuam no acolhimento, classificando riscos (do setor da emergência) a maioria já teria sofrido variados tipos de violência no ambiente laboral, até física. Busnello GF, et al. (2021), atentam para os profissionais da enfermagem que atuam na APS e Estratégia da Saúde da Família (ESF), os quais também 
atuam em condições muitas vezes inadequadas, sem suporte para atender a demanda que lhes procuram, e muitas vezes o processo de trabalho que enfrentam os expõem a sofrer violências diversas, gerando na equipe um sentimento de insegurança, tensão e medo.

Os estudos realizados por Martins BS e Pereira MC (2021), também destacam que a maioria das vítimas de violência ocupacional na enfermagem são do sexo feminino, sendo este fator relacionado a cultura da submissão, machismo, que apesar de todo processo de evolução feminina no âmbito do trabalho, busca pela igualdade e isonomia, ainda deixa suas marcas, pois, se apresentam ainda mais vulneráveis a esse tipo de violência. Além do perfil feminino, as mais jovens, com maior nível de escolaridade, são as vítimas mais incidentes, bem como o fato de estar iniciando (recém-contratado) ou ter pouca experiência no trabalho ser um indicativo de vulnerabilidade para o agressor (PEDRO DRC, et al., 2018).

A equipe de enfermagem em particular os enfermeiros ficam expostos a esse tipo de violência por estarem mais próximos aos pacientes e com isso são os primeiros a receberem tal manifestação, por motivo de insatisfação ou demora no atendimento, entre outros fatores que serão apresentados no presente estudo (TSUKAMOTO SAS, et al., 2019).

Ferreira SRS, et al. (2018) explica que, a preocupação gira em torno destas violências que, parecem se tornar rotineiras, causando muito mal ao corpo de enfermagem dos ambientes hospitalares, unidades de pronto atendimento e APS, na qual os mesmos são preparados para o cuidado, e agora, devido as precárias condições de trabalho, precisam se preparar para enfrentar usuários, gerentes, superiores violentos, pois, os agressores não são apenas os cidadão (usuário), e sim de outros trabalhadores, a própria equipe de trabalho e até mesmo a chefia, que agem violentamente de forma explícita e velada. Então, identificar essas situações que acometem esses profissionais é primordial para a prevenção e orientação de medidas e políticas públicas para evitar tal ato, pois esse tipo de violência afeta tanto a saúde e produtividade dos trabalhadores da saúde, os quais podem desenvolver outras doenças.

Apesar da violência laboral estar presente em todos os espaços hospitalares, o presente estudo está voltado para a APS, pois, os profissionais de saúde da atenção básica são os principais elos de acesso da população à saúde pública (BUSNELLO GF, et al., 2021).

Face ao exposto, o presente estudo tem como objetivo analisar o impacto deste tipo de violência sobre a saúde dos profissionais da enfermagem que atuam na APS. Os resultados podem oferecer informações importantes para que se intervenha nesta situação, a fim de melhorar as condições de trabalho dos referidos.

\section{MÉTODOS}

A metodologia empregada é de uma revisão integrativa da literatura, utilizada por seu potencial de organização, síntese de conhecimentos e identificação de lacunas que contribuem na análise crítica de um objeto de estudo proposto. Para isso, seguiu-se os seis passos para seu desenvolvimento: formulação da questão norteadora; busca na literatura; extração dos dados dos estudos selecionados; avaliação dos estudos; interpretação e síntese dos resultados; apresentação da revisão integrativa.

Para responder ao objetivo do presente estudo, formulou-se a seguinte questão norteadora: "Qual o impacto da violência ocupacional sobre a saúde dos enfermeiros (as) que atuam na APS?"

O levantamento bibliográfico tomou por base, dados da Literatura Latino-Americana e do Caribe em Acervo+, Ciências da Saúde (LILACS), Literatura Internacional em Ciências da Saúde (PUBMED), na Biblioteca Eletrônica Científica Online (Scielo) e Biblioteca Virtual em Saúde (BVS), o que possibilitou a busca por estudos publicados no cenário nacional.

Para a seleção dos estudos, optou-se pela inclusão de artigos científicos disponíveis na íntegra, em livre acesso, publicados no período entre 2016 a 2021, nos idiomas português, bem como aqueles que, após leitura do título e resumo, abordassem aspectos relacionados à temática do estudo. Foram excluídos os artigos duplicados, artigos de revisão, de reflexão/debates, monografias, dissertações, teses, comentários, editoriais e cartas. 
Os estudos elegíveis foram lidos na íntegra, a fim de incluir ou não, aqueles que conseguissem responder à questão norteadora. A interpretação e síntese dos resultados encontrados será apresentada em uma tabela contendo os autores, ano, local e periódico de publicação, bem como os principais resultados quanto aos impactos da violência ocupacional sobre saúde dos enfermeiros (as) que atuam na APS.

\section{RESULTADOS}

Foram encontrados 108 artigos nas bases de dados, dos quais em um primeiro momento, foram excluídos 82 artigos por não atenderem os seguintes critérios de inclusão: 8 estudos por estarem indisponíveis, 14 por estarem inferiores ao período previamente selecionado, 17 por estarem duplicados, 13 por serem artigos de revisão, 15 por serem teses, 15 por estarem em outro idioma.

Após a leitura de título e resumo, 26 artigos foram selecionados para leitura na íntegra, e 8 foram excluídos por não corresponderem ao propósito da pesquisa, o que possibilitou ao final, a inclusão de 18 artigos para serem analisados a fim de subsidiar a revisão de literatura (Figura 1).

Figura 1 - Fluxograma das etapas para a seleção dos artigos desta revisão integrativa, 2021.

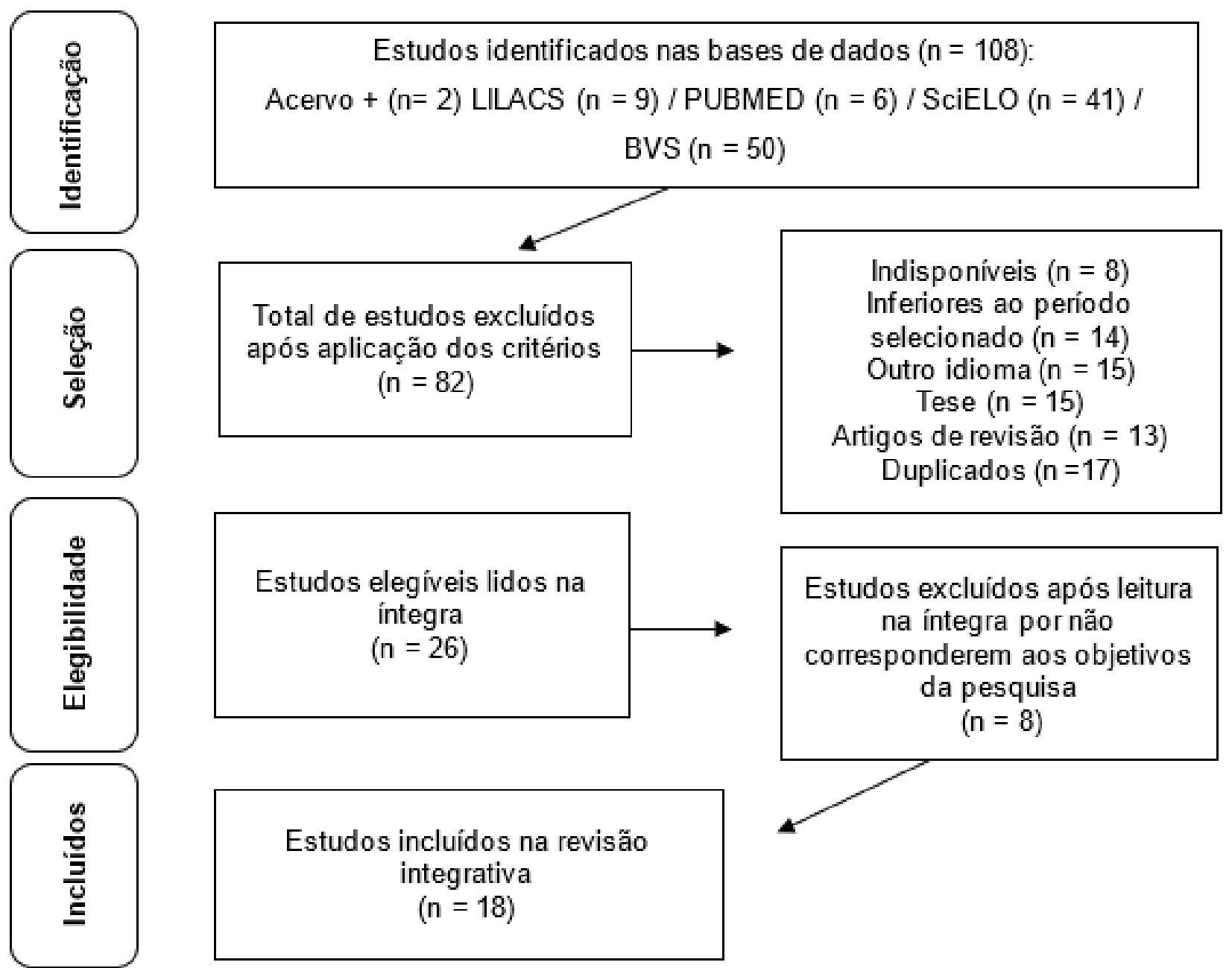

Fonte: Alexandre MG, et al., 2021.

Dos artigos incluídos na revisão, a maioria foi publicado no idioma português (99\%), e apenas um no idioma inglês (1\%), que foi traduzido. Quanto ao tipo de estudo, identificou-se majoritariamente, pesquisas do tipo transversais analíticos (33\%), transversais com abordagem qualitativa (28\%) / quantitativa (22\%) observacionais (11\%), Experimental (6\%). 
Quadro 1 - Características dos artigos incluídos neste estudo, de acordo com os autores, ano e periódico de publicação, tipo de estudo compreendendo os impactos da violência ocupacional sobre a rotina laboral de profissionais que atuam na APS.

\begin{tabular}{|c|c|c|c|}
\hline Autores e Ano & $\begin{array}{l}\text { Periódico de } \\
\text { publicação }\end{array}$ & $\begin{array}{l}\text { Tipo de } \\
\text { estudo }\end{array}$ & Vertente do artigo \\
\hline $\begin{array}{l}\text { BORDIGNON M e } \\
\text { MONTEIRO MI } \\
(2016)\end{array}$ & Rev Bras Enferm & $\begin{array}{l}\text { Transversal } \\
\text { qualitativo }\end{array}$ & $\begin{array}{l}\text { Violência no trabalho da Enfermagem: um } \\
\text { olhar às consequências }\end{array}$ \\
\hline $\begin{array}{l}\text { MEDEIROS PA, et } \\
\text { al. (2016) }\end{array}$ & $\begin{array}{l}\text { Revista Brasileira } \\
\text { de Ciências da } \\
\text { Saúde }\end{array}$ & $\begin{array}{l}\text { Transversal } \\
\text { quantitativo }\end{array}$ & $\begin{array}{l}\text { Condições de saúde entre Profissionais da } \\
\text { Atenção Básica em Saúde do Município de } \\
\text { Santa Maria - RS }\end{array}$ \\
\hline $\begin{array}{l}\text { SANTOS MS, et al. } \\
\text { (2017) }\end{array}$ & $\begin{array}{l}\text { Revista Brasileira } \\
\text { em Promoção da } \\
\text { Saúde }\end{array}$ & $\begin{array}{l}\text { Transversal } \\
\text { qualitativo }\end{array}$ & $\begin{array}{l}\text { O enfrentamento à violência no âmbito da } \\
\text { estratégia saúde da família: desafios para a } \\
\text { atenção em saúde }\end{array}$ \\
\hline $\begin{array}{r}\text { XAVIER } \\
(20\end{array}$ & Anais, UNIT & $\begin{aligned} \text { Tra } \\
\text { qu }\end{aligned}$ & $\begin{array}{l}\text { A Violência Sofrida pelo Enfermeiro no } \\
\text { Sistema de Saúde. }\end{array}$ \\
\hline $\begin{array}{l}\text { FREITAS } \\
\text { al. }(2\end{array}$ & $\begin{array}{l}\text { Revista Gaúcha de } \\
\text { Enf. }\end{array}$ & Transv & $\begin{array}{l}\text { A violência contra os profissionais da } \\
\text { enfermagem no setor de acolhimento com } \\
\text { classificação de risco }\end{array}$ \\
\hline $\begin{array}{l}\text { PEDRO DRC, et al. } \\
\qquad(2018)\end{array}$ & $\begin{array}{l}\text { Rev Saúde e } \\
\text { Debate }\end{array}$ & $\begin{array}{l}\text { Transversal } \\
\text { Analítico }\end{array}$ & $\begin{array}{l}\text { Violência ocupacional na equipe de } \\
\text { enfermagem: análise à luz do conhecimento } \\
\text { produzido }\end{array}$ \\
\hline $\begin{array}{l}\text { SILVA ACA, et al. } \\
(2018)\end{array}$ & Revista Diá & $\begin{array}{l}\text { Tra } \\
\text { qua }\end{array}$ & $\begin{array}{l}\text { A prática da violência voltada aos } \\
\text { profissionais da enfermagem }\end{array}$ \\
\hline $\begin{array}{l}\text { STURBE } \\
\text { et al. }\end{array}$ & Acta Paul Enferm. & $\begin{aligned} \text { Tral } \\
\mathrm{Ar}\end{aligned}$ & $\begin{array}{l}\text { Violência no trabalho em saúde da família: } \\
\text { estudo de métodos mistos }\end{array}$ \\
\hline $\begin{array}{r}\text { FONTA } \\
\quad(20\end{array}$ & Revista Vivências & Observacional & $\begin{array}{l}\text { A violência no cotidiano de trabalho da } \\
\text { enfermagem e os usos de si no } \\
\text { enfrentamento }\end{array}$ \\
\hline $\begin{array}{r}\text { SOARES E } \\
(201\end{array}$ & $\begin{array}{l}\text { Revista } \\
\text { Física \& } 1\end{array}$ & $\begin{aligned} & \text { Tral } \\
& \text { an }\end{aligned}$ & $\begin{array}{l}\text { Violência: a saúde dos trabalhadores de } \\
\text { enfermagem em risco }\end{array}$ \\
\hline $\begin{array}{l}\text { ROBAZZI MLCC, } \\
\text { et al. (2020) }\end{array}$ & $\begin{array}{l}\text { Brazilian Jo } \\
\text { health } \mathrm{R} \epsilon\end{array}$ & $\begin{array}{c}\text { Trai } \\
\text { qua }\end{array}$ & $\begin{array}{l}\text { Violência ocupacional antes e em tempos da } \\
\text { pandemia da covid-19: ensaio teórico e } \\
\text { reflexivo }\end{array}$ \\
\hline $\begin{array}{l}\text { JÚNIOR AMF, et } \\
\text { al. (2020) }\end{array}$ & $\begin{array}{r}\text { Revista } \\
\text { Ac } \\
\text { Enfer }\end{array}$ & $\begin{array}{l}\text { Transversal } \\
\text { Analítico }\end{array}$ & $\begin{array}{l}\text { Produção científica acerca do assédio moral } \\
\text { nas relações de trabalho de enfermagem no } \\
\text { Brasil }\end{array}$ \\
\hline $\begin{array}{l}\text { FERRE } \\
\quad \text { al. }(\end{array}$ & $\begin{array}{r}\text { Brazilian } \\
\text { he }\end{array}$ & Observacional & $\begin{array}{l}\text { enfermeiros durante } \\
\text { cação de risco }\end{array}$ \\
\hline $\begin{array}{l}\text { MARTINS BS e } \\
\text { PEREIRA MC } \\
(2021)\end{array}$ & $\begin{array}{l}\text { Rese } \\
\text { and } \mathrm{L}\end{array}$ & $\begin{array}{r}\text { Tran } \\
\text { An }\end{array}$ & Violência ocupacional na Enfermagem \\
\hline $\begin{array}{l}\text { TAVARES DS, et } \\
\text { al. (2020) }\end{array}$ & $\begin{array}{l}\text { Revista Eletrônica } \\
\text { Acervo Saúde }\end{array}$ & $\begin{array}{l}\text { Tranversal } \\
\text { qualitativo }\end{array}$ & $\begin{array}{l}\text { Aspectos relacionados } \text { à violência } \\
\text { ocupacional sofrida pelos profissionais de } \\
\text { enfermagem }\end{array}$ \\
\hline $\begin{array}{l}\text { SILVA, LO et al. } \\
\text { (2020) }\end{array}$ & $\begin{array}{l}\text { Revista Eletrônica } \\
\text { Acervo Saúde }\end{array}$ & $\begin{array}{l}\text { Tranversal } \\
\text { Analítico }\end{array}$ & $\begin{array}{l}\text { A violência sofrida pelos profissionais de } \\
\text { enfermagem no ambiente de trabalho }\end{array}$ \\
\hline $\begin{array}{l}\text { BUSNELLO GF, et } \\
\text { al. (2020) }\end{array}$ & $\begin{array}{l}\text { Revista Esc. Anna } \\
\text { Nery }\end{array}$ & Experimental & $\begin{array}{l}\text { Tipos de violência no trabalho da } \\
\text { enfermagem na Estratégia Saúde da Família }\end{array}$ \\
\hline $\begin{array}{l}\text { BARBOSA LCFS e } \\
\text { ARENAS MVS } \\
\text { (2020) }\end{array}$ & $\begin{array}{l}\text { Brazilian Journal of } \\
\text { Development }\end{array}$ & $\begin{array}{l}\text { sal } \\
0\end{array}$ & $\begin{array}{l}\text { Prevenção e combate à violência } \\
\text { ocupacional em profissionais de saúde }\end{array}$ \\
\hline
\end{tabular}

Fonte: Alexandre MG, et al., 2021.

\section{DISCUSSÃO}

Diante dos estudos selecionados, pode-se constatar que há uma vasta literatura relacionada a violência ocupacional sofrida pela enfermagem, todavia, voltada especificamente a atenção básica ainda e limitada, de 
forma que este estudo é composto de 18 artigos sobre a temática. Dentre os estudos selecionados, 05 apontaram que os profissionais da enfermagem da APS vem sendo expostos constantemente à violência no trabalho, sendo mais predominante a violência verbal, devido à falta de informação do usuário e a forma como se comunicam, muitas vezes desrespeitosa com o atendente, devido a insatisfação com o agendamento, bem como, a postura profissional de alguns profissionais que não atuam de forma humanizada, não acolhendo bem os pacientes, sendo estas falhas associadas a atenção primária (FREITAS RJM, et al., 2017; XAVIER AS, et al., 2017; FONTANA, RT, 2019; BARBOSA LCFS e ARENAS MVS, 2021, SILVA ACA, et al., 2018).

O segundo tipo de violência mais prevalente na APS é a violência física, com destaque ao fato de que os profissionais de enfermagem menos experientes foram mais acometidos da violência ocupacional, talvez por ainda não apresentarem segurança total em seus procedimentos ou por demonstrarem desinformação e insegurança na hora de atender o paciente, além de não estarem acostumados e preparados para a forma grosseira com que muitos usuários tratam os profissionais da enfermagem (BORDIGNON M e MONTEIRO MI, 2016).

As formas de violência física mais relatadas foram agressões como chutes, tapas, empurrões, atirar objetos, murros, etc. (PEDRO DRC, et al., 2019). Muitas das vezes, a violência ocorre por falta de um bom acolhimento, com classificação de risco, o qual busca atender todo o público, os quais requerem um atendimento responsável e resolutivo, que muitas vezes as condições de trabalho da enfermagem não pode oferecer, deixando os acompanhantes desesperados e fora de si (FERREIRA JC, et al., 2020).

Evidencia-se também violência psicológica e assédio moral (TAVARES DS, et al., 2020). Xavier AS, et al. (2017) também apontam violência sexual e ameaças presentes no cotidiano destes profissionais, mesmo porque a grande parte dos profissionais são do sexo feminino, sendo esta uma questão cultural no país, em diversos setores da sociedade. Martins BS e Pereira MC, (2021) ainda destacam a discriminação racial entre as violências sofridas por estes profissionais na APS, na qual os usuários buscam ofender os profissionais, na tentativa de diminuir os profissionais associando sua cor. Nesse sentido, Busnello GF et al. (2021) afirmam em seus estudos que, a discriminação racial, sobretudo a trabalhadores da enfermagem que apresentam pela parda ou negra, os quais sentem reduzidas a satisfação na realização do seu ofício, em decorrência desses fatos.

Entres os agressores citam-se os pacientes, seus acompanhantes, os próprios colegas de trabalho e, muitas vezes seus superiores (TAVARES DS, et al., 2020; SILVA LO, et al., 2021). O assédio moral geralmente é causado pelos superiores, se enquadrando também em violência institucional, sendo que o mesmo e considerado uma combinação de diferentes naturezas de atos violentos, com capacidade de gerar uma crise de identidade e intenso sofrimento, acarretando transtornos psicológicos que podem resultar em sequelas, que variam de um indivíduo para outro, desde crises de choro a depressão, diminuição da libido aos distúrbios digestivos, ideias de suicídio ao alcoolismo (BARBOSA LCFS e ARENAS MVS, 2021).

De acordo com Soares BB, et al., (2019), os danos causados podem ser revelados de forma imediata ou não, bem como, podem ser passageiros ou durar anos, de forma que, leve o trabalhador da enfermagem a desenvolver depressão, pânico, suicídio e outros modos de autoflagelação. Concordam Bordignon $\mathrm{M}$ e Monteiro MI (2016); Medeiros PA, et al. (2016) e Tavares DS, et al. (2020) que os impactos têm causado aos profissionais da enfermagem que desenvolvem seus trabalhos na APS, problemas de saúde como: danos físicos, insegurança, ansiedade manifestações emocionais, transtornos e distúrbios psíquicos

Da mesma forma, estudos apontaram que a violência no trabalho pode afetar negativamente 0 desempenho do trabalhador, pois, as consequências e tal ato são imensuráveis e acabam influenciando a vida não apenas de um indivíduo, mas, muitas vezes, de um grupo familiar e as relações sociais (BORDIGNON M e MONTEIRO MI, 2016; JÚNIOR AMF, et al., 2020). Os impactos podem ser expressados na desilusão pela profissão, observada no distanciamento do profissional na forma de se relacionar com os colegas de trabalho e com os usuários, levando esses profissionais ao desânimo, depressão e a frustração profissional (XAVIER AS, et al., 2017). Durante a pandemia covid 19, foram relatados impactos como: desejo de abandonar a profissão (ROBAZZI MLCC, et al., 2020). 
Soma-se aos desgastes físicos, emocionais apresentados pelos profissionais de enfermagem na APS, o fato de que os mesmos têm uma rotina de trabalho desgastante, logo, os mesmos dispõem de pouco tempo para o lazer e para aderir a hábitos que cultuem a saúde mental e física, de forma a comprometer sua qualidade de vida (MEDEIROS PA, et al., 2016). Frente ao que foi visto nesses estudos, Medeiros PA, et al. (2016) disserta que, a violência ocupacional ocorre na APS, pois, mesmo prestando um serviço que não tem muito aparato tecnológico, implica em outras exigências técnicas, o que leva a necessidade de capacitações contínuas, políticas públicas de valorização a classe da enfermagem, direcionada sobretudo para cada tipo de complexidade em que os profissionais estejam lotados, seja na Atenção Básica, como na baixa e alta complexidade (MEDEIROS, PA et al., 2016).

Deve-se considerar que, na saúde, é preciso se cuidar não somente do que é atendido, mas do que atende, só assim há eficácia no atendimento (ROBAZZI MLCC, et al., 2020). Dessa forma o impacto da violência projetada sobre a enfermagem na APS pode ser amortecido, por meio de melhoria nas condições de trabalho, fortalecimento da APS, a fim de que as Equipes de Saúde melhorem o processo de comunicação com a comunidade, e consiga criar sistemas de atendimento mais adequados a cada realidade, fortalecendo o elo das equipes com os usuários acompanhados.

Há necessidade de maior sensibilização e humanização no acolhimento, o que poderia ser fomentado durante a formação destes enfermeiros (as) nas Universidades, ou promovidas capacitações voltadas para humanização, acolhimento e sensibilização dessa classe, além de campanhas contra o racismo, assédio moral e pela valorização das mulheres na sociedade, em especial as profissionais da enfermagem que tem (SANTOS MS, et al., 2017).

Fontana RT (2019) e Júnior AMF, et al. (2020), alertam para que sejam criadas e fomentadas estratégias de enfrentamento à essa forma de violência, como medidas de notificação, pois as mesmas contribuem para que este fenômeno seja divulgado, e em cima dos números de casos possa-se elaborar estratégias e ações para reduzir esses casos, agregando valor à saúde dos enfermeiros (as).

Portanto verifica-se a necessidade de uma maior abordagem, debates e atenção ao assunto, para que o poder público, assim como a sociedade dediquem maior vigilância ao problema e reúnam esforços na busca de medidas eficazes que visem prevenir e reprimir ações esse tipo de violência, considerada como doença social que traz complicações severas para suas vítimas (PEDRO DRC, et al., 2019).

\section{CONSIDERAÇÕES FINAIS}

Pode-se verificar que há similaridades nos estudos relacionados ao tipo de violência ocupacional mais prevalente, que é a verbal, seguida da física, psicológica, por meio de ameaças, assédio moral, e até violência sexual e discriminação racial, advinda de pacientes, seus acompanhantes e gerentes ou superiores. Podem afetar o trabalhador, e se a mesma for contínua pode abalar psicológica, emocional e fisicamente o mesmo. Sugere-se o implemento de políticas organizacionais, campanhas nas mídias sociais que sejam aptas a enfrentar a violência ocupacional que, como visto nesse estudo está associada a práticas racistas, machistas e infundadas, pois, trazem danos à dignidade e à integridade dos profissionais da enfermagem, levando-os ao adoecimento psíquico e físico e consequentemente diminuindo sua qualidade laboral, impactando para o próprio usuário.

\section{REFERÊNCIAS}

1. BARBOSA LCFS, ARENAS MVS. Prevenção e combate à violência ocupacional em profissionais de saúde. Brazilian Journal of Development, 2021; 7(6): 60158-60176.

2. BERNARDES MLG. Violência laboral entre trabalhadores de enfermagem. Rev Bras Med Trab., 2020; 18(3): 250257.

3. BORDIGNON M, MONTEIRO MI. Violência no trabalho da Enfermagem: um olhar às consequências. Revista Brasileira de Enfermagem, 2016; 69(5): 996-999.

4. BUSNELLO GF, et al. Tipos de violência no trabalho da enfermagem na Estratégia Saúde da Família. Escola Anna Nery, 2021; 25(4): e20200427. 
5. JÚNIOR AMF, et al. Produção científica acerca do assédio moral nas relações de trabalho de enfermagem no Brasil. Revista Eletrônica Acervo Enfermagem, 2020; 3: e2742.

6. FERREIRA JC, et al. Violência vivenciada por enfermeiros durante acolhimento com classificação de risco. Braz. J. Hea. Rev, 2020; 3(6): 15812-15825.

7. FERREIRA SRS, et al. A complexidade do trabalho do enfermeiro na Atenção Primária à Saúde. Rev. Bras. Enferm. 2018, 71: 704-709.

8. FONTANA RT. A violência no cotidiano de trabalho da enfermagem e os usos de si no enfrentamento. Vivências, 2019; 16(30), 99-114.

9. FREITAS RJM, et al. A violência contra os profissionais da enfermagem no setor de acolhimento com classificação de risco. Revista Gaúcha de Enfermagem, 2017; 38(3): e62119.

10. MARTINS BS, PEREIRA MC. Occupational Violence in Nursing. Research, Society and Development, 2021; 10(7): e50910717246.

11. MEDEIROS PA, et al. Condições de saúde entre profissionais da atenção básica em saúde do município de Santa Maria - RS. Revista Brasileira de Ciências da Saúde, 2016; 20(2): 115-122.

12. PEDRO DRC, et al. Violência ocupacional na equipe de enfermagem: análise à luz do conhecimento produzido. Saúde em Debate [online], 2017; 41(113): 618-629.

13. ROBAZZI MLCC, et al. Violência ocupacional antes e em tempos da pandemia da covid-19: ensaio teórico e reflexivo. Braz. J. Hea. Rev, 2020; 3(6): 19042-19064.

14. SANTOS MS, et al. O enfrentamento à violência no âmbito da estratégia saúde da família: desafios para a atenção em saúde. Revista Brasileira em Promoção da Saúde, 2017; 30(2): 229-238.

15. SILVA LO, et al. A violência sofrida pelos profissionais de enfermagem no ambiente de trabalho. Revista Eletrônica Acervo Saúde. 2021; 13(8): 1-11.

16. SILVA ACA, et al. A prática da violência voltada aos profissionais da enfermagem. Revista Diálogos Interdisciplinares, 2018; 7(2): 100-108.

17. SOARES BB. Violência: a saúde dos trabalhadores de enfermagem em risco. Revista Saúde Física \& Mental- SFM, 2019; 7(1): 60-73.

18. STURBELLE ICS, et al. Violência no trabalho em saúde da família: estudo de métodos mistos. Acta Paulista de Enfermagem [online], 2019; 32(6): 632-641.

19. TAVARES DS, et al. Aspectos relacionados à violência ocupacional sofrida pelos profissionais de enfermagem. Revista Eletrônica Acervo Saúde, 2021; 13(2): e5881.

20. TEIXEIRA NL, et al. Desafios do enfermeiro no gerenciamento de conflitos dentro da equipe de enfermagem. Rev. Adm. Saúde, 2018; 18(73): 130-138.

21. TSUKAMOTO SAS, et al. Violência ocupacional na equipe de enfermagem: prevalência e fatores associados. Acta Paul Enferm, 2019; 32(4): 425-432.

22. XAVIER AS, et al. A Violência sofrida pelo Enfermeiro no Sistema de Saúde. Anais. International Nursing Congress, 2017; 1(1): 9-12. 